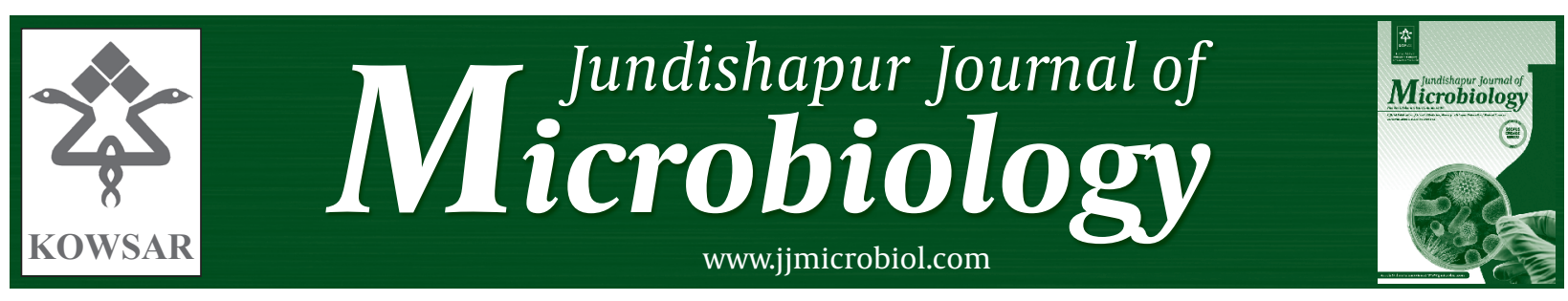

\title{
Frequency of Mupirocin Resistant Staphylococcus aureus Strains Isolated From Nasal Carriers in Hospital Patients in Kermanshah
}

\author{
Parviz Mohajeri ${ }^{1^{*}}$, Babak Gholamine ${ }^{2}$, Mansour Rezaei ${ }^{3}$, Yazdan Khamisabadi ${ }^{4}$ \\ ${ }^{1}$ Department of Microbiology, Faculty of Medicine, Kermanshah University of Medical Sciences, Kermanshah, IR Iran \\ 2 Department of Pharmacology and Toxicology, Faculty of Pharmacy, Kermanshah University of Medical Sciences, Kermanshah, IR Iran \\ ${ }^{3}$ Social Development and Health Promotion Research Center, Biostatistics Department, Faculity of Public Health, Kermanshah University of Medical Sci- \\ ences, Kermanshah, IR Iran \\ ${ }^{4}$ Student Research Committee, Kermanshah University of Medical Sciences, Kermanshah, IR Iran
}

\begin{tabular}{|c|c|}
\hline A R T I C L E I N F O & A B S T R A C T \\
\hline $\begin{array}{l}\text { Article type: } \\
\text { Original Article }\end{array}$ & $\begin{array}{l}\text { Background: : Staphylococcus aureus is a major nosocomial pathogen world wide. Mupi- } \\
\text { rocin plays a crucial role in strategies designed to control outbreaks of S. aureus. } \\
\text { obiectives: } \text { The aim of this study was to determine the freauency of mupirocin resis- }\end{array}$ \\
\hline $\begin{array}{l}\text { Article history: } \\
\text { Received: } 19 \text { Jan } 2012 \\
\text { Revised: 10 Mar } 2012 \\
\text { Accepted: } 4 \text { Apr } 2012\end{array}$ & $\begin{array}{l}\text { tance in S. aureus strains isolated from nasal carriers among the hospitalized patients at } \\
\text { Kermanshah Hospital, Iran. } \\
\text { Patients and Methods: A total of } 174 \text { S. aureus isolates (sensitive and resistant to methi- } \\
\text { cillin) were collected from the nasal anterior nares of hospitalized patients. All isolates }\end{array}$ \\
\hline $\begin{array}{l}\text { Keywords: } \\
\text { Staphylococcus aureus } \\
\text { Mupirocin }\end{array}$ & $\begin{array}{l}\text { hibitory concentration (MIC) was determined by an E-test and they were also analyzed } \\
\text { by a PCR for the presence of ileS-1 and ileS-2 genes. } \\
\text { Results: Utilizing the disc diffusion agar method, E-test and PCR, all of the S. aureus } \\
\text { strains tested were susceptible to mupirocin. In this study, the range of mupirocin MICs } \\
\text { was determined to be between } 0.064 \text { and } 4 \mu \mathrm{g} / \mathrm{ml} \text {. There was a significant association } \\
\text { between MIC observed and multi-drug resistant (MDR) carriage (P value 0.04), and resis- } \\
\text { tance to oxacillin ( } P \text { value } 0.004) \text {. } \\
\text { Conclusions: This is a report of an initial survey of mupirocin resistance in S. aureus, in } \\
\text { Kermanshah where the use of mupirocin is still limited. Perhaps the sensitivity of all } \\
\text { isolates to mupirocin in this study is due to the less common usage of this antibiotic, } \\
\text { especially in the form of nasal and site sample collections. }\end{array}$ \\
\hline
\end{tabular}

Published by Kowsar Corp, 2012. cc 3.0.

- Implication for health policy/practice/research/medical education:

Mupirocin resistance has been associated with failure to clear MRSA from nasal carriage patients.

Please cite this paper as:

Mohajeri P, Gholamine B, Rezaei M, Khamisabadi Y. Frequency of Mupirocin Resistant Staphylococcus aureus Strains Isolated From Nasal Carriers in Hospital Patients in Kermanshah.Jundishapur J Microbiol. 2012;5(4):560-63. DOI: 10.5812/jjm.4199

\footnotetext{
* Corresponding author: Parviz Mohajeri, Department of Microbiology, Faculty of Medicine, Kermanshah University of Medical Sciences, Shirudi shahid Blvd, Daneshgah St, P.O. Box: 67148-69914, Kermanshah, IR Iran. Tel:+98-8314274618, Fax:+98-8314276477, E-mail: p_mohajeri@yahoo.com

DOI:10.5812/jjm.4199

(C) 2012 Ahvaz Jundishapur University of Medical Sciences; Published by Kowsar Corp.

This is an Open Access article distributed under the terms of the Creative Commons Attribution License(http://creativecommons.org/licenses/by/3.0), which permits unrestricted use, distribution, and reproduction in any medium, provided the original work is properly cited.
} 


\section{Background}

Staphylococcus aureus has been recognized as the main etiological agent and the most frequent microorganism in community-acquired and hospital-acquired infections (1). Nasal colonization with S. aureus is common and it is an important step in the pathogenesis and spread of S. aureus infections, these strains provide a reservoir for infection in other sites such as; surgical-site and bloodstream infections. In certain subgroups, such as; frequently hospitalized people, senile and immune compromised patients, colonization with $S$. aureus occurs more frequently (2). Currently, the health problems associated with this microorganism have become more serious due to an increasing incidence of methicillin-resistant S. aureus (MRSA) (3). As an antibiotic, mupirocin (pseudomonic acid A) is an analogue of isoleucine that inhibits protein synthesis by competitively binding to the enzyme isoleucyl-tRNA synthetase. It was first introduced in the UK as one of the most effective topical antibiotic that is active against gram-positive pathogens, as well as some gram-negative bacteria and it is used for the eradication of $S$. aureus in the nasal carriage $(4,5)$.

of the two mupirocin-resistant phenotypes, the lowlevel resistant strain (MIC8-256 $\mu \mathrm{g} / \mathrm{ml}$ ) is more common with a point mutation by the isoleucyl-tRNA synthetase gene (ileS-1) for the target enzyme, and a high-level of mupirocin resistance (MIC $\geq 512 \mu \mathrm{g} / \mathrm{ml}$ ), from the acquisition of a plasmid carrying a new gene, ileS-2 or (mupA), it encodes an alternate isoleucyl-tRNA synthetase $(6,7)$. Nasal carriage therapy with mupirocin ointment appears to be effective in reducing the onset and severity of infections at surgical sites (1).

\section{Objectives}

At present, the prevalence of these resistant organisms from the nasal carriages of patients in the Kermanshah Hospital, which is the largest hospital in western Iran, is still unknown.

\section{Patients and Methods}

\subsection{Specimen Collection and Bacterial Detection}

Nasal swabs were taken five times by rotating a sterile cotton swab, moistened with sterile saline, in the vestibule of both anterior nares of hospitalized patients in different wards (ICU, CCU, surgery, internal medicine, gynecology, infection, heart, pediatric, infants and hemodialysis) of the Kermanshah Hospital which is the largest hospital in western Iran, between October 2009 and August 2010 (6, 7). Trypticase soy broth (TSB) was used as a transport medium. Swabs were transported in Amies medium and processed within two hours of collection. The swabs were put directly onto mannitol salt agar (Merck, Germany) and sent to the laboratory and incubated at $35^{\circ} \mathrm{C}$ in a humidified incubator for $48 \mathrm{~h}$. Strains that produced yellow colonies on the MSA plate, were subcultured on a blood agar plate (Merck, Germany), for further characterization.

The staphylococcal isolates were identified by conventional methods, e.g. colonial morphology, gram staining characteristics, production of catalase, coagulase tube method using rabbit plasma, DNase in tube tests and other biochemical tests (8). A total of 174 non-repetitive S. aureus isolates (sensitive and resistant to methicillin) were collected.

\subsection{Antibiotic Susceptibility Testing}

Screening for methicillin resistance was determined by a Kirby-Bauer disk diffusion test, according to the guidelines published by the Clinical and Laboratory Standards Institute. Using a $30 \mu \mathrm{g}$ cefoxitin disk (MAST, UK), the disk was placed on Mueller-Hinton agar (Merck, Germany) and incubated for $24 \mathrm{~h}$ at $35^{\circ} \mathrm{C}$ following CLSI guidelines (9).

In determining resistance to mupirocin as the first step, all isolates were screened by the disk diffusion method using a $5 \mu \mathrm{g}$ mupirocin paper disk (MAST). An isolate with inhibition zones $\geq 14 \mathrm{~mm}$ around the disk were designated as sensitive, but these discs failed to distinguish between low level and high level resistance (9, 10). To analyze the sensitivity patterns of the mupirocin strains more precisely, MICs for S. aureus isolates to mupirocin were assessed using E-test $®$ mupirocin strips (AB-BIODISK, Solna, Sweden) according to the manufacturer's instructions. The E-test strip was applied onto each plate of Mueller-Hinton agar that was inoculated with a suspension of isolates to the optical density of a 0.5 McFarland standard with sterile forceps. Following incubation at $35^{\circ} \mathrm{C}$ for $24 \mathrm{~h}$. E-test MIC values were read by the operator at the point where the bottom of the inhibition zone intersected with the E-test strip. Strains were considered to be susceptible if the MIC value was $\leq 4 \mathrm{mg} / \mathrm{L}$ and levels of mupirocin resistance were defined as low-level with MIC 8-256 mg/l and high-level with MIC $\geq 512 \mathrm{mg} / \mathrm{L}$ (7). For both MSSA and MRSA, we defined multi-drug resistant (MDR) isolates as those that were resistant to 3 different antibiotics, i.e. co-trimoxazole, ciprofloxacin and erythromycin (11).

\subsection{Polymerase Chain Reaction (PCR)}

All of the strains were analyzed for the presence of the iles -1 and the ileS-2 (mupA) gene by boiling the bacterial cells and PCR $(12,13)$. To extract the DNA, the isolated staphylococci were cultured on blood agar (Merck, Germany) for 24 hours. Subsequently, a loop full of bacterial cells from each sample were removed and resuspended in $250 \mu \mathrm{l}$ of sterile distilled water and the suspension incubated in a $90^{\circ} \mathrm{C}$ heat block for $15 \mathrm{~min}$. Centrifugation followed $(7500 \times \mathrm{g}, 5 \mathrm{~min})$ and the supernatant containing the staphylococcal DNA extract was transferred into 
new test tubes and frozen for later PCR amplification. Five microlitres of the extracted DNA was transferred to $20 \mu \mathrm{l}$ of the PCR amplification mix consisting of; $2.5 \mu \mathrm{l}$ of $10 \mathrm{X}$ buffer, $1.5 \mathrm{mM}$ of $\mathrm{MgCl}_{2}, 1.5 \mathrm{U}$ of Taq polymerase, $1.25 \mu \mathrm{l}$ of dNTPs and $1.5 \mu$ of each primer. To identify point mutations of the ileS-1 gene, the 690-bp product was amplified using a primer pair $1 \mathrm{mr1}$ (5'-GTA AAT CTT TAG GTA ATG TGA TTG TAC-3') and lmr2 (5'-TCT TCT TTA ACA TGT GGT GTA TGA GA-3') (13). To detect the ileS-2 gene (mupA gene), a 456-bp region in the ileS-2 gene was amplified by PCR, using a primer pair Mup1 (5'-TAT ATT ATG CGA TGG AAG GTT GG-3') and Mup2 (5'-AAT AAA ATC AGC TGG AAA GTG TTG-3') (14). Cycling parameters were $94^{\circ} \mathrm{C}$ for $5 \mathrm{~min}$ followed by 30 cycles, denaturation at $94^{\circ} \mathrm{C}$ for $30 \mathrm{~s}$, annealing at $52^{\circ}$ $\mathrm{C}$ for $30 \mathrm{~s}$ and extension at $72^{\circ} \mathrm{C}$ for $30 \mathrm{~s}$, and a final $5 \mathrm{~min}$ incubation at $72^{\circ} \mathrm{C}$. Following PCR amplification, the amplification (PCR) products were analyzed by agarose gel electrophoresis (1× Tris-Boric acid-EDTA, $1.2 \%$ agarose, $75 \mathrm{~V}$, $70 \mathrm{~min}$ ) stained with ethidium bromide and the amplicons were visualized using a UV light box.

S. aureus ATCC (25923) was used as a reference strain for a Mup quality control during susceptibility testing and DNA S. aureus jj1 from Marcia Giambiagi-deMarval (Brazil) were mupirocin resistant and used as the reference strain quality control for PCR (12).

\subsection{Statistical Analysis}

The data was entered in Microsoft Access XP software and exported into SPSS statistical software, version 16.0, which was used for the data analyses. The categorical data were compared using a chi-squared test or Levene's test. All $P$ values were two-sided with $P<0.05$ being considered significant.

\section{Results}

Of the 174 patients studied, 93 (53.4\%) were male and 81 (46.6\%) were female, ranging in age from one to 84 and from one to 83 years, respectively. Mean age was 39.47 \pm 25.92 and $45.26 \pm 24.37$ for males and females, respectively. The MIC of the individuals was not associated significantly with age. ( $P$ value 0.859 ) With the disc diffusion agar method, all of the $174 \mathrm{~S}$. aureus strains tested were susceptible to mupirocin. Single PCR of each gene was conducted for all of the isolated, but none of the strains contained iles-1 and ileS-2.

In this study, the E-test range of mupirocin MICs was determined to be between 0.064 and $4 \mu \mathrm{g} / \mathrm{mL}$. A total of 108 (62.2\%) strains had MICs less than $1 \mu \mathrm{g} / \mathrm{mL}$ and 66 (37.8\%) strains had MICs more than $1 \mu \mathrm{g} / \mathrm{mL}$. Of the 174 isolates, $92(52.8 \%)$ were found to be methicillin resistant and 82 (47.2\%) were methicillin susceptible. Their mean MIC was $1.33 \pm 1.38$ and $0.81 \pm 0.95$ for the MRSA and MSSA strains respectively. In the Levene's test, the MIC of the individuals was significantly associated with resistance to oxacillin ( $P$ value 0.004$)$. Of the 174 isolates, 99 (56.9\%) were multi- drug resistant. In the Levene's test, their mean MIC was $1.24 \pm 1.31$ and $0.89 \pm 1.06$ respectively for the MDR positive and MDR negative isolates ( $P$ value 0.04 ).

\section{Discussion}

Based on our findings, none of the S. aureus isolates (MRSA and MSSA) were mupirocin resistant and none of the demographic characteristics of the carriers or antibiotic resistance patterns or even the source of the isolates (hospital/ community acquired) showed any determinant role in mupirocin sensitivity. The MIC of 16 (9.2\%) of the strains was $4 \mu \mathrm{g} / \mathrm{mL}$ which is very close to a low level resistance $(8 \mu \mathrm{g} / \mathrm{mL})$. The emergence of mupirocin resistance among $S$. aureus isolates has been clearly defined in many parts of the world at different frequencies: Spain $11.3 \%$, USA $13.2 \%$, Trinidad Tobago $26.1 \%$, China 6.6\%, India $6 \%$, Turkey $45 \%$ and Korea $5 \%$, however, it does appear to be increasing worldwide $(2,4,5,10,14-16)$.

In the two reports from Iran, the prevalence of such resistant strains was $2.7 \%$ and $0 \%(1,7)$. It can be assumed that the absence of resistant strains in our study may be related to two factors: the rare use of mupirocin as an empiric therapy in Kermanshah and lack of clinical samples. In Iran, although mupirocin is not applied to eliminate $S$. aureus nasal colonization in patients as part of infection control measures, it is infrequently used for the treatment of skin infections. Rapid resistance to mupirocin occurs among strains of S. aureus isolated from hospitals. Therefore, monitoring for mupirocin resistance in S. aureus, especially in MRSA, is necessary to evaluate the usefulness of mupirocin in both the treatment of staphylococcal infections and infection control.

The observed MIC of 4 in this study, therefore, recommends a continuous surveillance plan in our area. To date, mupirocin has not yet been used for the eradication of $S$. aureus in nasal carriers in our hospital. But a local application of mupirocin ointment has been shown to eliminate MRSA nasal colonization in both patients and hospital staff. The full susceptibility of $S$. aureus to mupirocin observed in this study indicates that it is effective for the treatment of S. aureus infections in our hospital. It seems that antibiotic use led to the emergence of resistant strains $(10,16)$. However, there are other studies which have reported the isolation of mupirocin-resistant S. aureus from patients who had not taken mupirocin (5, $7,15)$.

Mupirocin-resistant strains were often resistant to other antimicrobial agents. For example there is a relationship between methicillin resistance and resistance to mupirocin, but in this study such a relationship has not been proven (14). However, MIC appears to be significantly greater among MRSA than in MSSA strains ( $P$ value 0.004 ). Our study showed that $80 \%$ of the strains with MIC $4 \mu \mathrm{g} / \mathrm{mL}$ were multidrug-resistant ( $P$ value 0.04 ). All of the studied strains showed the same results in the disc 
diffusion agar method, E-test and PCR. So, we think that there is no significant difference between these methods in the detection of mupirocin resistance.

In conclusion, the present study revealed that our studied S. aureus strains were completely sensitive to mupirocin and the prevalence of mupirocin resistant $S$. aureus strains in the nasal carrier patients was similar to the prevalence that was reported by the other study in our country $(1,7)$. Further studies in different regions of Iran will show the level of this similarity for consideration in national infection control programs. Nonetheless, the need for continuous surveillance remains important. Detecting the introduction of mupirocin-resistant strains, especially in hospital fields which have shown higher levels of resistance to mupirocin and multiple reports of higher rates of resistance to mupirocin in methicillin resistance isolates, is the rationale for such surveillance.

\section{Acknowledgements}

This work was performed in partial fulfillment of the requirements for Pharmacy student's thesis (Yazdan Khamisabadi) and we acknowledge with thanks Kermanshah University of Medical Sciences.

\section{Financial Disclosure}

None declared.

\section{Funding Support}

The study was financially supported by the Kermanshah University of Medical Sciences for grant 89156.

\section{References}

1. Askarian M, Zeinalzadeh A, Japoni A, Alborzi A, Memish ZA. Prevalence of nasal carriage of methicillin-resistant Staphylococcus aureus and its antibiotic susceptibility pattern in healthcare workers at Namazi Hospital, Shiraz, Iran. Int J Infect Dis. 2009;13 (5):e241-7.

2. Jones JC, Rogers TJ, Brookmeyer P, Dunne WM, Jr., Storch GA, Coopersmith $\mathrm{CM}$, et al. Mupirocin resistance in patients colonized with methicillin-resistant Staphylococcus aureus in a surgical intensive care unit. Clin Infect Dis. 2007;45 (5):541-7.
3. Sousa-Junior FC, Silva-Carvalho MC, Fernandes MJ, Vieira MF, Pellegrino FL, Figueiredo AM, et al. Genotyping of methicillin-resistant Staphylococcus aureus isolates obtained in the Northeast region of Brazil. Braz J Med Biol Res. 2009;42 (10):877-81.

4. Daskalaki M, Otero JR, Chaves F. Molecular characterization of resistance to mupirocin in methicillin-resistant Staphylococcus aureus isolates in a tertiary hospital in Spain. J Antimicrob Chemother. 2009;63 (4):826-8.

5. Liu QZ, Wu Q, Zhang YB, Liu MN, Hu FP, Xu XG, et al. Prevalence of clinical meticillin-resistant Staphylococcus aureus (MRSA) with high-level mupirocin resistance in Shanghai and Wenzhou, China. Int J Antimicrob Agents. 2010;35 (2):114-8.

6. Lim KT, Hanifah YA, Mohd Yusof MY, Thong KL. Prevalence of mupirocin resistance in methicillin-resistant Staphylococcus aureus strains isolated from a Malaysian hospital.Jpn J Infect Dis. 2010;63 (4):286-9.

7. Saderi H, Owlia P, Habibi M. Mupirocin resistance among Iranian isolates of Staphylococcus aureus. Med Sci Monit. 2008;14 (10):BR210-3.

8. Halablab MA, Hijazi SM, Fawzi MA, Araj GF. Staphylococcus aureus nasal carriage rate and associated risk factors in individuals in the community. Epidemiol Infect. 2010;138 (5):702-6.

9. Performance Standards for Antimicrobial Susceptibility Testing: 17th Informational Supplement. Journal [serial on the Internet]. 2007; 27(1): Available from: http://www.microbiolab-bg.com/ CLSI.pdf.

10. Sareyyupoglu B, Ozyurt M, Haznedaroglu T, Ardic N. Detection of methicillin and mupirocin resistance in staphylococcal hospital isolates with a touchdown multiplex polymerase chain reaction. Folia Microbiol (Praha). 2008;53 (4):363-7.

11. Pathak A, Marothi Y, Iyer RV, Singh B, Sharma M, Eriksson B, et al. Nasal carriage and antimicrobial susceptibility of Staphylococcus aureus in healthy preschool children in Ujjain, India. BMC Pediatr. 2010;10:100.

12. de Oliveira NE, Cardozo AP, Marques Ede A, dos Santos KR, Giambiagi-deMarval M. Interpretive criteria to differentiate low- and high-level mupirocin resistance in Staphylococcus aureus. J Med Microbiol. 2007;56 (Pt 7):937-9.

13. Park DW, Kim MJ, Yang JA, Jeong HW, Sohn JW, Chun BC. Risk factors for isolation of low-level mupirocin-resistant versus -susceptible methicillin-resistant Staphylococcus aureus from patients in intensive care units. J Infect. 2007;54 (4):337-42.

14. Yun HJ, Lee SW, Yoon GM, Kim SY, Choi S, Lee YS, et al. Prevalence and mechanisms of low- and high-level mupirocin resistance in staphylococci isolated from a Korean hospital. J Antimicrob Chemother. 2003;51 (3):619-23.

15. Gadepalli R, Dhawan B, Mohanty S, Kapil A, Das BK, Chaudhry R, et al. Mupirocin resistance in Staphylococcus aureus in an Indian hospital. Diagn Microbiol Infect Dis. 2007;58 (1):125-7.

16. Orrett FA. The emergence of mupirocin resistance among clinical isolates of methicillin-resistant Staphylococcus aureus in Trinidad: a first report.Jpn J Infect Dis. 2008;61 (2):107-10. 